\title{
LA ADQUISICIÓN DEL ESPAÑOL L2/E COMO PROCESO DE SUBJETIVACIÓN. UNA PROPUESTA DESDE EL INTERACCIONISMO ESTRUCTURAL
}

\author{
The acquisition of Spanish as S/FL as a subjectivazing process. A proposal from \\ structural interactionism
}

\author{
María Virginia Bruzzo* \\ Universidad Nacional del Nordeste \\ virginiabruzzo@gmail.com
}

\author{
Palabras clave \\ adquisición de segundas \\ lenguas; \\ ELE; \\ proceso de subjetivación; \\ interacción; \\ interaccionismo \\ estructural
}

\section{Keywords \\ second language \\ acquisition; \\ SFL;}

subjectivazing process;

interaction;

structural interactionism

\section{Resumen}

En este artículo presentamos los primeros resultados de una investigación exploratoriadescriptiva, en la que estudiamos la adquisición del español en cinco estudiantes de intercambio, procedentes de Brasil, Dinamarca, Francia y Bélgica, en dos cursos de español como lengua extranjera (ELE), uno en la Facultad de Humanidades de la UNNE y otro en un instituto privado de idiomas, en Resistencia y Corrientes (Argentina), respectivamente. Sostenemos, acorde con nuestro marco, el interaccionismo estructural (Lemos, 2000a, 2000b, 2002; Desinano, 2009, 2018), que el sujeto pasa, al igual que el niño, por un proceso de subjetivación en la lengua extranjera, que consiste en la captura del sujeto por el lenguaje, a través del habla del Otro-docente, compañeros $\mathrm{u}$ otras personas-, entendida como instanciaciones de la lengua. En este proceso de subjetivación el sujeto establece relaciones con el lenguaje que se traducen en posiciones estructurales, no superables, con un polo dominante. En la primera posición el polo dominante es el Otro, retornan en el habla del alumno fragmentos del habla del Otro; en la segunda posición, es la lengua, emergen en el discurso estructuras latentes del español; y en la tercera, es el sujeto, que se hace consciente de su propia habla, se escucha, se corrige y reformula.

\begin{abstract}
This article presents the first results of an exploratory-descriptive research, in which it is studied the acquisition of Spanish by five exchange students, from Brazil, Denmark, France and Belgium, in two courses of Spanish as a foreign language (SFL): in the Faculty of Humanities, UNNE, and in a private language institute, in Resistencia and Corrientes (Argentina), respectively. It is argued, according to the framework of structural interactionism (Lemos, 2000a, 2000b, 2002; Desinano, 2009, 2018), that the subject, like the child, undergoes a subjectivizing process in the foreign language, which consists of the capture of the subject by le langage, through the parole of the Other-teacher, classmates or other people-, considered as instantiations of la langue. In this subjectivazing process, the subject establishes relations with le langage that are considered structural positions that cannot be overcome, with a dominant pole. In the first position the dominant pole is the Other, fragments of the Other's parole return in the student's parole; in the second position, it is the langue, latent structures of Spanish emerge in the discourse; and in the third position, it is the subject, who becomes aware of his own parole, listens to it, corrects and reformulates it.
\end{abstract}




\section{Consideraciones iniciales: el campo de la adquisición de segundas lenguas (ASL)}

Con apenas sesenta años de historia, la adquisición de segundas lenguas (ASL) -denominación que incluye las lenguas extranjeras o, simplemente, adicionales- es un campo relativamente reciente pero aun así altamente productivo. Su breve historia, no siempre libre de disputas internas, estuvo marcada por varias etapas caracterizadas por diferentes corrientes o enfoques teóricos que intentaron dar respuesta al fenómeno de la adquisición de segundas lenguas. Especialmente en los últimos treinta años, hubo un florecimiento de múltiples estudios en este campo interdisciplinar, influido por teorías de otras disciplinas, como la lingüística, la psicología, la antropología, la psicolingüística, la sociolingüística y los estudios sobre la adquisición de lenguas primeras, entre otras (Ellis, 2020).

Ellis (2020) realizó una breve presentación de las etapas y teorías propias de esta disciplina. Los inicios en los 60 y 70 estuvieron marcados por estudios sobre aprendientes en contextos naturales que ponían en cuestionamiento las perspectivas conductistas de enseñanza y el método audiolingual. La segunda etapa, en los años 80, se caracterizó por una expansión de las teorías: desde las centradas en las hipótesis de transferencias lingüistica y pragmática, la influencia de la gramática universal y las primeras teorías centradas en el input y la interacción (Krashen, 1985; Long, 1983; y Swain, 1985 en Ellis, 2020). En los 90 se desarrollaron las siguientes etapas: la tercera, con mayor preeminencia de las teorías cognitivistas (Schmidt, 1990; Tomlin y Villa, 1994, Ellis, 1994 y DeKeyser, 1998 en Ellis, 2020); y la cuarta, con el giro hacia los estudios centrados en los aspectos sociales (Firth y Wagner, 1997; Block, 2003; y Norton, 2000 en Ellis, 2020) y los estudios socioculturales (Lantolf, 2000 y Swain, 2006 en Ellis, 2020). Finalmente, arribaron en la quinta etapa, los recientes desarrollos de la teoría de los sistemas dinámicos complejos de Larsen-Freeman y los estudios centrados en el multilingüismo (Cook, 1992; May, 2013 y Ortega, 2009, 2019 en Ellis, 2020).

Estos sesenta años parecen estar marcados por el debate entre la necesidad de buscar una teoría fuerte y "única” que explique el fenómeno para hacer de la ASL una disciplina pura, y la continuidad del carácter aplicado de esta área de estudio en las aulas de enseñanza de lenguas extranjeras, lo que implica la continuación del desarrollo de la convivencia de variadas teorías, relacionadas con distintos aspectos en la enseñanza de L2/E (Ellis, 2020). Sin entrar en este debate, y con el fin de contribuir al campo en estudio, presentaremos aquí los primeros resultados de una investigación de alcance exploratorio-descriptivo en la que nos propusimos estudiar el fenómeno de la adquisición de una L2/E en el marco del interaccionismo estructural de Claudia de Lemos (1986, 2000a, 2000b, 2002). El estudio se centra en la adquisición del español como lengua extranjera de cinco estudiantes de español de distintas nacionalidades en dos centros de estudios, en la Facultad de Humanidades de la Universidad Nacional del Nordeste (UNNE) y en un instituto privado de idiomas, en las ciudades de Resistencia y Corrientes (Argentina). Finalmente, en cuanto a los resultados de esta investigación, esperamos que constituyan un aporte a la enseñanza de E/LE y que puedan ser aplicados en el futuro a estudios sobre la adquisición de otras lenguas. 


\section{Motivaciones y búsqueda de posibles respuestas: el interaccionismo estructural}

La motivación del estudio de la adquisición de segundas lenguas se fundamenta en la idea de que, conociendo mejor los procesos internos y externos de adquirir una lengua extranjera, se pueden desarrollar modos de enseñanza más efectivos -sean en contextos áulicos alejados de los lugares en los que la L2 se habla o en inmersión-. Es por ello que, movidos por la necesidad de investigaciones en ELE por la inclusión de la UNNE en el Consorcio ELSE para ser sede del examen CELU, ${ }^{1}$ y por nuestra experiencia en el aula, cada vez más demandante por el creciente número de alumnos de intercambio - provenientes de Brasil, especialmente, y de otros países no hispanohablantes-, es que nos propusimos realizar un estudio sobre la adquisición del español como lengua segunda o extranjera.

A diferencia, quizá, de lo que se esperaría habiendo tantas corrientes teóricas activas en el área de la ASL, decidimos realizar un estudio exploratorio sobre la adquisición del español de nuestros alumnos de intercambio, teniendo como base la teoría sobre adquisición del interaccionismo estructural desarrollada en Brasil por Claudia de Lemos (1986, 2000a, 2000b, 2002). Tomamos esta decisión debido a que los estudios que se estaban llevando a cabo en nuestra institución, en relación a las prácticas letradas de los alumnos ingresantes de la Facultad de Humanidades, tenían en cuenta como marco teórico las investigaciones de Norma Desinano (2009, 2018), continuadoras del interaccionismo estructural. Conjeturamos, por tanto, considerando la experiencia en el aula de ELE, que los conceptos de esta teoría sobre las relaciones entre el sujeto y el lenguaje, en el proceso de adquisición de lengua materna, podrían explicar también el proceso de adquisición del ELE.

Claudia de Lemos (2000a, 2000b, 2002) inicia los estudios que originarían su teoría interaccionista estructural, a partir de la crítica que realiza sobre (a) las teorías interaccionistas que no logran explicar cómo la interacción modifica y amplía los recursos iniciales del niño (Lemos, 1986) y (b) la concepción de lenguaje como un objeto divisible en partes que pueden ser aprendidas y dominadas como una herramienta, habiendo suficientes muestras de lo contrario, considerando la heterogeneidad y la imprevisibilidad de los errores durante el proceso de adquisición (Lemos, 1986, 2000a). Es así como, en sus investigaciones, habiendo encontrado un posible marco en el psicoanálisis lacaniano y la lingüística saussureana, busca describir cómo la interacción modifica y amplía los recursos iniciales del niño (Lemos, 1995, 2000a, 2000b, 2002). En esta teoría propone que el niño pasa por un proceso de subjetivación, que consiste en la captura del niño por el lenguaje, a través del habla de la madre, entendida como instanciaciones de la lengua, hasta la constitución del sujeto en el lenguaje (Lemos, 2000, 2002). Más precisamente, el niño pasa por distintas posiciones estructurales no superables en las que hay un polo dominante, determinado por las relaciones que el niño establece con el lenguaje. En la primera posición, el polo dominante es el habla del Otro, madre-cuidador, el niño imita el habla del Otro recogiendo fragmentos de ella. En la segunda, el polo dominante es la lengua, ya que en el habla emergen estructuras latentes de la lengua -y no simples fragmentos- con una fluctuación de usos correctos e incorrectos. En la tercera, el polo dominante es el sujeto, el niño se vuelve consciente de su propia habla, es por ello que aparece la escucha que le permite corregirse y reformularse (Lemos,

1. El Certificado de Español Lengua y Uso (CELU) es el examen de proficiencia del español ofrecido por la República Argentina a través del Consorcio ELSE, compuesto por universidades nacionales argentinas. 
2000a, 2000b, 2002). De ese modo, no se llega a un conocimiento omnipotente sobre la lengua, ni a una subjetividad producto de las identificaciones del babla de la madre; el sujeto funciona en y por el lenguaje (Lemos, 2000a).

Esta teoría fue retomada por Norma Desinano (2009) quien observó que sus estudiantes universitarios establecían las mismas relaciones con el discurso académico que el niño con la lengua materna. En este sentido, postuló que el estudiante universitario pasa por un proceso de subjetivación al enfrentarse por primera vez a los discursos académico-científicos (Desinano, 2009); proceso que se concibe como una constante en la vida del sujeto ante cada nueva instancia en la que se enfrenta con discursos desconocidos (Desinano, 2009, 2018). La autora propuso, retomando las posiciones lemosianas, dos polos situados en un continuum, el polo de la fragmentariedad y el polo de continuidad, que señalan las relaciones que el estudiante establece con el discurso académico-científico en sus producciones escritas. En el polo de la fragmentariedad, los textos se caracterizan por rupturas que fragmentan la escritura, se trata de fallos, errores, lapsus y hápax. En un primer momento, el estudiante, como el niño en la primera posición, retoma fragmentos de discursos otros, de los textos de referencias y/o de los profesores, a manera de una amalgama; el docente debe reconstruir estos fragmentos para darles sentido (Desinano, 2009). En un segundo momento, en los textos emergen cadenas latentes de los discursos otros que deben ser repuestos por el docente, es por ello que hay un anclaje, una dependencia, en las fuentes, aunque ya no se trata de una simple amalgama de fragmentos (Desinano, 2009). Finalmente, más cercanos al polo de la continuidad, un polo ideal sin errores, se ubican los escritos en los que se percibe la escucha en el estudiante, esto se debe a que este se vuelve consciente de su propio discurso, lo que le permite corregirse y reformularse; los textos son versiones propias de las teorías presentadas en los textos de referencias y por el profesor en el aula (Desinano, 2009).

Como señalamos, en nuestra investigación, en la que exploramos otros conceptos que nos den respuestas a los fenómenos que observábamos en nuestros estudiantes, retomamos esta propuesta teórica. Según lo analizado hasta ahora, propusimos que la adquisición del ELE se da como un proceso de subjetivación, en el que el estudiante es capturado por el español, o, en otros términos, el estudiante es alienado (Desinano, 2009) por la lengua, en el sentido de que se constituye como sujeto en el español (Wingeyer y Bruzzo, 2019; Wingeyer, Bruzzo y Angelina, 2019; Bruzzo y Wingeyer, 2019). Según notamos, los estudiantes de ELE establecen las mismas relaciones con el español que el niño con su lengua materna y que el estudiante universitario con los discursos académicos. En este artículo nos proponemos presentar los primeros resultados sobre el proceso de subjetivación y la función de la interacción en el mismo.

\section{La puesta en marcha: métodos y herramientas}

\subsection{Sujetos en estudio}

Para esta parte de nuestra investigación, observamos dos cursos de ELE durante los años 2019 y 2020: 
(i) Uno, dictado durante septiembre y noviembre de 2019 en la Facultad de Humanidades de la UNNE (Resistencia), a K de 24 años, estudiante avanzada de Ingeniería Mecánica, y a J de 26 años. Al finalizar el curso, las dos estudiantes brasileñas del programa de intercambio ESCALA rindieron el examen CELU -con un resultado de un Avanzado Bueno para K y de un Intermedio Muy Bueno para J-.

(ii) Otro, dictado en un instituto privado (Corrientes) a los siguientes estudiantes: N, danesa de 16/17 años, quien había finalizado el folkeskole del sistema de educación danés; T, francés de 17/18 años, quien había finalizado el lycée del sistema de su país; y E, belga de 17/18 años, estudiante del equivalente al nivel secundario en su país. Este último curso duró de fines septiembre a principios de diciembre de 2019. Por otra parte, $\mathrm{N}$ tomó clases particulares presenciales de español hasta julio de 2020, y desde abril de 2020, luego de su retorno a Dinamarca, clases virtuales.

\subsection{Recolección de datos}

Para la recolección de datos se recurrió, en primer lugar, a la observación participante, la docente-investigadora observó y registró, en un cuaderno de campo y mediante grabaciones de audio, las interacciones entre los estudiantes y la docente en clases, en ambos cursos. Estas grabaciones fueron luego transcriptas siguiendo el sistema de transcripción utilizado por la cátedra de Lingüística II de la carrera de Fonoaudiología de la Universidad Nacional del Rosario. ${ }^{2}$ Además, se recogieron 50 muestras de producciones escritas, realizadas en distintos momentos del período de cursado, correspondientes a las resoluciones de actividades presentadas a través de artefactos pedagógicos (Camblong, Di Módica y Alarcón, 2012), que sirvieron también para la recolección de datos.

\subsection{Método de análisis}

En esta investigación exploratoria analizamos las instancias de adquisición en las clases de ELE y en las producciones escritas de alumnos extranjeros. Para la descripción y análisis de los corpus oral y escrito, procesos que siguen en desarrollo, recurrimos al análisis del discurso de la línea francesabrasilera (Lemos, 2000a, 2000b, 2002; Desinano, 2009). Identificamos en las producciones fenómenos lingüísticos-discursivos (Lemos, 2000a, 2000b, 2002; Desinano, 2009) que indicarían la transición por las posiciones en la subjetivación del estudiante y cómo la interacción modificó y amplió sus recursos.

\section{El proceso de subjetivación en español comolengua extranjera: primeros resultados}

En las siguientes secciones presentamos los resultados de la observación, descripción y análisis de los corpus oral y escrito antes mencionados. Como se verá, definimos las mismas posiciones del proceso de subjetivación del interaccionismo estructural, aunque identificamos algunas situaciones específicas dentro de la segunda y tercera posición que no corresponden de modo directo con lo descripto por

2. Para detalles sobre este sistema de transcripción, véase Báez, M. (2000). 
Lemos o Desinano en las posiciones, pero indudablemente hay un paralelismo entre lo que describimos y lo desarrollado por ambas autoras.

\subsection{Primera posición}

En la primera posición, el polo dominante es el habla del Otro. Al igual que en el niño (Lemos, 2000a, 2002), retornan en el habla del estudiante fragmentos del habla del Otro, sean el docente, compañeros u otras personas con las que haya interactuado. Se podría decir que se trata de un proceso de metonimia (Lemos, 2000a, 2002). El habla es fragmentaria en los niveles fonológico, morfológico y sintáctico.

Diferenciamos, al igual que Lemos (2002), dos momentos específicos. En un primer momento, los fragmentos que retornan en el habla del estudiante son altamente fragmentarios, mientras que, en un segundo momento, los fragmentos son estructuralmente más complejos.

En esta primera posición, el Otro, el docente o determinados compañeros, toma el rol de intérprete (Lemos, 2000a, 2002) que "restringe las indeterminaciones"3 (Lemos, 2000a, p. 171) del discurso del alumno. El docente o los compañeros reconstruyen los fragmentos y devuelven el significante (o las cadenas de significantes) al estudiante, quien al reconocerlo como propio hace que la interacción avance. Esta interpretación pareciera ser crucial para el movimiento a la siguiente posición. El cuerpo del aprendiente entendido como corps pulsionnel, al igual que el del niño, demanda interpretación constante.

\subsubsection{Primer momento}

En este primer momento la fragmentariedad en el habla de los estudiantes es mayor. Se observa, como fue indicado antes, que hay un "retorno en el habla" (Lemos, 2002, p. 58) del alumno "de parte de los enunciados usados" (Lemos, 2002, p. 58) por el Otro -profesor, compañero u otras personas-en instancias de interacción anteriores; situaciones que son reconocibles por el estudiante. El Otro toma el rol de interpretar al alumno y de significarlo.

El fragmento I que aquí reproducimos corresponde a una clase del 14 de octubre de 2019 en el instituto privado, en la que estaban presentes $\mathrm{N}$ y E. En este momento de la clase, las alumnas habían hecho algunas preguntas sobre cómo expresar en español ciertas emociones. $\mathrm{N}$ específicamente consultó sobre cómo decir "I am excited", y retornan en su habla fragmentos de habla de un Otro, de una situación de interacción similar a la que se dio en la clase: conversar sobre cómo expresar en español ciertas emociones. Sin embargo, debido al nivel de fragmentación a nivel fonológico de estos fragmentos de habla, la profesora no reconoció inmediatamente las cadenas de significantes a las que remitía. Se dio así una situación de interpretación para restringir "las indeterminaciones" (Lemos, 2000a, p. 171).

N: How do you say that (?) I always say estoy [Iməvfonadə] ${ }^{4}$ It doesn't mean I'm nervous it means that I'm looking forward right (?)

3. Todas las traducciones del inglés y portugués son propias.

4. En este caso tuvimos que recurrir al alfabeto fonético internacional para intentar mostrar de manera más fiel el habla de la aprendiente. 
E: ah excited (!)

N: how do you say excited (?)

Profesora: entusiasmada -lo escribió en el pizarrón-

$\mathrm{N}$ : what (?) oh but when I say estoy [imofonado] I say that I'm nervous (?)

Profesora: no I don't understand what (?)

E: nervous

Profesora: yeah

$\mathrm{N}$ : [Iməvfonadə] because when I'm like

E: [imozionada] it means that you're really like emotional

Profesora: AH emocionada

$\mathrm{N}$ : no no what I want to say

E: ella quiere [dezer] que ella está [nervioza]

Profesora: sí que ella

$\mathrm{N}$ : no I don't want to say that I want to say that I'm excited like when I find something really interesting it's like estoy /Iməozonada/

E: +++

Profesora: imaginado (?)

$\mathrm{N}$ : yeah (?)

Profesora: em

$\mathrm{N}$ : it seems I said something weird

Profesora: imagi

N: no no ee [emərfonada]

Profesora: $\mathrm{mm}$

N: I'm e /

Profesora: emocionada emocionada -lo escribió en el pizarrón- e mo cio na da

$\mathrm{N}$ : e mo cio na da is that something that you use (?)

Profesora: sí es un sinónimo de esta palabra -señaló entusiasmada-

(Fragmento I - 14102019)

Como se observa, la profesora, con ayuda de $\mathrm{E}$, interpreta los enunciados de $\mathrm{N}$, quien hasta no reconocer en el habla de la profesora las cadenas de significantes que retornan fragmentariamente en su habla, la interacción no avanza. Además, como se ve, $\mathrm{N}$ muestra una aparente incredulidad ante el hecho de que "emocionada" sea equivalente a "excited" debido a su similitud con la palabra "emotional", que tiene un sentido diferente en inglés. Se observa así en N un choque entre significantes, en su subjetivación en el inglés, su L2, y las del español, concebido como una alteridad, a la que, justamente por ello, presenta una cierta resistencia. Es así que ocurre en principio una confusión entre los significantes que retornan en su habla, "estoy emocionada", los significantes que la simbolizan, "I'm excited", y los enunciados de la profesora "estoy entusiasmada". No fue hasta que la profesora dijo "emocionada", dos veces a un ritmo "normal" de habla y otra vez más marcando cada sílaba, y anotó el significante en el pizarrón, que $\mathrm{N}$ pudo reconocer(se) en el habla del Otro. El avance en la interacción se puede identificar en la pregunta "is that something that you use?", que denota esta incredulidad de la que hablábamos y que muestra una resistencia a este singificante. A través de esta pregunta $\mathrm{N}$ intentaba dar sentido a esta palabra como un posible equivalente de la emoción que intentaba expresar.

Finalmente, en lo que respecta a la presencia de la L2 de la alumna en su discurso, observamos que la imposibilidad de hacerse sujeto en esta lengua desconocida lleva a la estudiante a recurrir a 
una lengua en la que sí se ha constituido como tal, en particular, en estas cadenas de significantes que le eran en ese momento totalmente ajenas y no así con otras situaciones en las que había una mayor predominancia de fragmentos de habla otra en sus enunciados. ${ }^{5}$ En este sentido, conjeturamos que, como sostienen otros enfoques como el multilingual (Ortega, 2009) -aunque desde otra perspectiva-, recurrir a las lenguas en las que se constituyeron (o están en proceso de constituirse) como sujetos puede ser verdaderamente útil para el proceso de captura, ya que es en este constante devenir de relaciones de diferenciación, resistencia y alienación que el sujeto se constituye en el lenguaje.

\subsubsection{Segundo momento}

Por su parte, el segundo momento se caracteriza por una "mayor extensión y complejidad de los fragmentos que migran" (Lemos, 2002, p. 58) del Otro al estudiante. En este caso presentamos el fragmento II que corresponde a la clase del 16 de diciembre en el instituto privado en la que estaban $\mathrm{N}$ y T. En este caso, estaban trabajando sobre el verbo soler en pretérito imperfecto, como $\mathrm{N}$ no recordaba su significado, entre $\mathrm{T}$ y la profesora intentaron explicárselo. Luego de que la profesora expusiera en líneas generales el uso del verbo y escribiera su conjugación en presente, sucedió la siguiente situación:

Profesora: ejemplo / solemos ir a la costanera / entendés (?)

$\mathrm{N}$ : - con cara pensativa- sí

Profesora: me podés dar un ejemplo (?)

N: sí / por ejemplo / solemos ir al shopping con mis amigas yo pregunto a mi mamá

T: y qué significa?

$\mathrm{N}$ : que yo pido permiso

Profesora: ah / no / no es una pregunta / es para un hábito / una rutina / suelo ir al gimnasio todos los días quiere decir que es algo que normalmente hago todos los días / suelo comer chipacitos en la merienda

T: es algo que haces siempre

$\mathrm{N}$ : ah (!) entiendo / yo suelo comer helado / siempre

Profesora: yo suelo / vos solés / tú sueles / él suele / es una rutina

N: ah -anot-a yo sue / lo / vos sóles / él sole

Profesora: él suele

N: él suele / nosotros / solemos (?) / ellos suelen

Profesora: ellos ellas ustedes suelen / sí

N: ujum

T: entiendes (?) es algo que hacés siempre

$\mathrm{N}$ : sí una rutina

Profesora: un hábito

T: sí / por ejemplo / vos sales el fin de semana puede decir

Profesora: podés o puedes decir

$\mathrm{T}$ : puede decir / yo suelo el fin de semanaa / salgoo / con T

$\mathrm{N}$ : ujum

Profesora: salir

$\mathrm{T}$ : a salir

5. Ver Bruzzo, M. V. y Wingeyer, H. (2020a; 2020b). 
Profesora: no no / suelo salir

$\mathrm{T}$ : ah ah (!) suelo salir suelo salir

$\mathrm{N}$ : um / qué es la palabra en inglés o / no hay (?)

Profesora: eeh sí se puede decir

$\mathrm{N}$ : can we (?)

Profesora: no no // no es exactamente lo mismo pero es similar / we are used to go out on the weekends /

$\mathrm{N}: \mathrm{mmm}$

T: pero depende / puede decir suelo / sa-lir / con T el fin de semana

$\mathrm{N}$ : ah (!) entonces no es una pregunta

T: eso es every every weekend you go out

Profesora: no no no / no es una pregunta

$\mathrm{N}: \mathrm{ah}$

Profesora: es una afirmación / yo suelo salir los fines de semana con T

$\mathrm{N}$ : ah (!) ok ok / then it's ++ / suelo / suelo comer una pizza en la costanera después escuela

T: y qué significa (?)

$\mathrm{N}$ : I use to /

T: -miró a la profesora-

Profesora: -risas- lo va a traducir

$\mathrm{N}$ : - confundida-

Profesora: decilo de otra manera

$\mathrm{N}:+++$ ah / siempre estoy después de escuela

T: estás en una pizzería

$\mathrm{N}$ : -asintió-

(Fragmento II - 16122019)

En este caso lo destacable de la interacción es que, por una parte, las intervenciones de T trajeron situaciones reconocibles por $\mathrm{N}$, lo que ayudó a la asociación del significante con estas situaciones (Bruzzo y Wingeyer, 2020a y 2020b). Por otra parte, destacamos las construcciones metonímicas de los enunciados de $\mathrm{N}$ en relación con los enunciados de la profesora (Bruzzo y Wingeyer, 2020a y 2020b), así cuando la profesora dijo "suelo salir a la costanera", $\mathrm{N}$ dijo "suelo salir al shopping"; y cuando la primera dijo "suelo comer chipacitos”, la alumna, "suelo comer helado". Como señalábamos más arriba esta metonimia es constitutiva de la primera posición, lo que diferencia este momento con el anterior, sin embargo, es la complejidad de los fragmentos que retornan.

\subsection{Segunda posición}

En la segunda posición el polo dominante es la lengua ya que emergen en el habla del estudiante estructuras latentes del sistema del español. A diferencia de la posición anterior, lo que caracteriza a esta no es la metonimia sino la metáfora: los procesos metafóricos (Lemos, 2002) se refieren a los procedimientos por los cuales en el habla del estudiante emergen las regularidades de la lengua, ya que construyen enunciados por similitud. Justamente por esto y al no tener una consciencia sobre su propia habla, hay una fluctuación entre enunciados correctos e incorrectos (por ejemplo, al emerger las regularidades del tiempo verbal, pueden no reconocer las irregularidades en ella). Al igual que para Lemos, el error caracteriza a esta posición. Esto se debe a que la relación de interacción es entre el sujeto y su lengua. Es por ello, además, que no escucha la diferencia entre su discurso y el del Otro, por lo que, aunque este restrinja las indeterminaciones de los enunciados incorrectos y señale la diferencia, 
el sujeto es impermeable a la diferencia entre su discurso y el del Otro. Como resultado, el estudiante puede (i) no darse por aludido ante el señalamiento; (ii) volver a repetir el "error" luego de que se lo corrija; o (iii) corregir momentáneamente su discurso para luego, volver a producir enunciados "incorrectos".

En esta posición no identificamos momentos diferenciables, pero sí situaciones que consideramos características de esta posición. Una se trata de la emergencia de estructuras latentes, es decir, situaciones en las que aparecen en el discurso del estudiante estructuras reconocibles del español descoladas -de fragmentos de habla procedentes de situaciones conocibles-y resignificadas en nuevas instanciaciones. La otra se trata de situaciones de inconsistencias entre usos correctos e incorrectos, en las que en una sola sesión se produce la alternancia de enunciados correctos e incorrectos de una misma estructura.

\subsubsection{Emergencia de estructuras latentes}

El caso del fragmento III, se trata de una situación en la que J y K estaban revisando junto a la profesora una actividad que consistía en el rellenado de huecos con la conjugación en pretérito imperfecto o indefinido del verbo indicado, según correspondía. Consideramos que esta es una muestra de la emergencia de estructuras latentes ya que da señales de regularidades de las estructuras en el babla de estas estudiantes. Además, es de notar que las mismas aprendientes señalan la no consciencia sobre estas estructuras, como si se aprendieran, según sus propias palabras, por "osmosis” [sic].

$\mathrm{J}$ : cuando entramos tenía que esperar porque la recepcionista no estaba (?)

Profesora: teníamos (J: Sí) el tiempo está bien pero tiene que ir en plural.

$\mathrm{K}$ : yo puse tuvimos existe eso (?) -risas-

Profesora: - risas- Sí de existir existe. / También puede ser esa opción

$+++$

J: y después de unos minutos nos / dijo ++ y lo peor de todo es que no tenía más habitaciones disponibles -risas-

K y Profesora: -risas-

K: puede ser tenían (?)

Profesora: puede ser ambos tenia o tenían / tenían es como más impersonal / bueno en realidad ambos / sí ambos se pueden usar de igual manera

$\mathrm{K}: \mathrm{mm}$

J: ella llamó a varios hoteles de la ciudad hasta que con / ...

Profesora: consiguió

K: consiguió

J: escribí cierto pero no

Profesora: sin la tilde (?)

$\mathrm{J}$ : no no pensaba que era correcto

[...]

Profesora: bien (!) bastante bien

J: sí pero bastante instinto instintivo porque yo escribí consiguió y fui pero ni séporqué

-risas-

$++$

$\mathrm{K}$ : por osmosis

-risas-

(Fragmento III - 29102019) 


\subsubsection{Inconsistencias entre usos correctos e incorrectos}

El fragmento $I V$ corresponde a una clase particular de N. Este fragmento es constitutivo de la segunda posición ya que como vemos se observan cadenas de significantes que se descolocan y resignifican en nuevas instanciaciones, y no ya simples fragmentos del habla del Otro. Notamos la inconsistencia entre usos correctos e incorrectos en la utilización de los verbos "ser" y "estar". Vemos una corrección momentánea luego de que la profesora le indicara la forma correcta del enunciado emitido, en "era bien" / "estaba bien", sin embargo, esta corrección no se debe a una consciencia sobre el error ya que inmediatamente después vuelve a utilizar el verbo "ser" en un caso en el que debía emplear "estar" (“fui mejor”).

N: Igual / Igual como creo que / cuando ee vive en el país ee sin mucho inglés / comoo y eee escuche ee escucho (?)

$[\ldots]$

Profesora: o vos / ee en este caso ee / se escucha (!) se escucha el

$\mathrm{N}$ se escucha

Profesora: el inglés eso querías decir (?)

$\mathrm{N}$ : sí sí- se escucha se escucha / no / el español español todo el tiempo en el televisor ooo

Profesora: sí

$\mathrm{N}$ : en ee -miró hacia la ventana-

Profesora: en la calle

$\mathrm{N}$ : en la calle sí / ee se lee lea / to read

Profesora: se lee

$\mathrm{N}$ : sí se lee / y la radio

Profesora: sí

$\mathrm{N}$ : sí es imposible no aprender algo

Profesora: sí mejoraste mucho

$\mathrm{N}$ : sí / también en en Brasil

Profesora: ujum

N: tenía un bien amigo de México /

Profesora: un buen amigo

N: un buen amigo de México que no babla

Profesora: ujum

$\mathrm{N}$ : habla (!) en inglés pero no muy bien entonces / hablábamos / en en español

Profesora: ujum súper

$\mathrm{N}$ : ee / pero -risa- también su español era muy difícil

Profesora: -risa-

N: pero también porque bablaba muy rápido / pero / era bien

Profesora: estaba bien

$\mathrm{N}$ : estaba bien para mi español

Profesora: súper (!)

$\mathrm{N}$ : ujum -sonríe- / pero él me dijo que en el comenso o al comenso(?) en el viaje

Profesora: en el principio o al comienzo

$\mathrm{N}$ : en el principo del viaje fui mejor que en el fin del viaje porque en (!) el fin del viaje

Profesora: ah claro

$\mathrm{N}$ : porque en el fin hacía un mes que estaba en Argentina

(Fragmento IV - 20022020) 


\subsection{Tercera posición}

En la tercera posición el polo dominante es el sujeto, lo que caracteriza a esta posición es la consciencia del propio discurso. El estudiante escucha su propia habla, ya que como sostiene Lemos (2002) en relación al niño, retomando a Freud, el sujeto que habla y el que escucha son distintos. A diferencia de la posición anterior, el estudiante construye sus enunciados siendo consciente de la diferencia, lo que le permite escuchar los enunciados erróneos y corregirlos. Además, dada la consciencia en el propio discurso, también el sujeto es capaz de reconocer sutilezas de la lengua como juegos de palabras, ironías, chistes, etc.

En la interacción con el Otro, docente o compañero, es el desconcierto del estudiante, frente a los cuestionamientos o preguntas sobre el discurso, lo que lleva a estimular el extrañamiento ante su propio discurso, es decir, a escucharse, hasta llegar a situaciones de escucha sin intervención del Otro.

Al igual que en la posición anterior, identificamos distintas situaciones que van de un señalamiento directo del error por parte del Otro a una escucha sin que otro lo indique. Por último, mostramos una situación de reconocimiento de un juego de palabras en la interacción.

\subsubsection{Corrección con indicación directa del Otro del error y la forma correcta}

El fragmento $V$ corresponde a una clase en octubre en el curso en la UNNE, K y la profesora estaban leyendo la producción escrita que aquella había realizado. A continuación, un fragmento del texto y la muestra de diálogo:

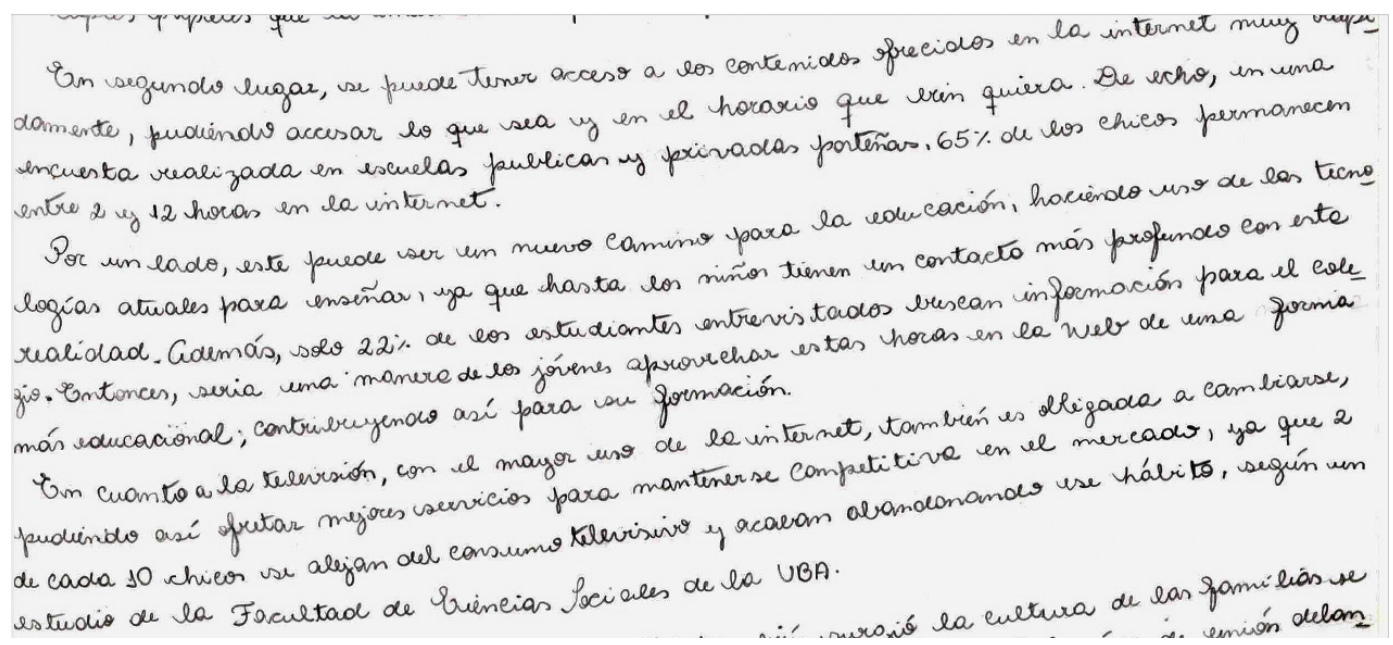

Imagen $1 . \mathrm{K}$ fragmento de escritura

En este caso, mientras avanzaba en la lectura, la profesora corregía el trabajo, correcciones que $\mathrm{K}$ reconoció, produciéndose un proceso de extrañeza ante cada error (Bruzzo y Wingeyer, 2020b).

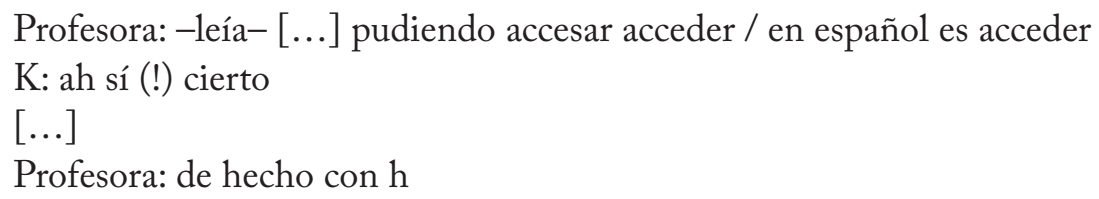


K: ah (!) no sé qué pasó ahí -risas-

$[\ldots]$

Profesora: acá / ofretar es

$\mathrm{K}$ : ofreee

Profesora: ofrecer

K: ah sí (!)

(Fragmento V-29102019)

Pareciera que la lectura del Otro motiva la extrañeza ante la diferencia entre la forma "incorrecta" y la "correcta", escucha que no había logrado en su lectura propia antes de entregar el trabajo. Este reconocimiento de la diferencia es lo que nos hace pensar que esta es una muestra de la transición de la segunda a la tercera posición. Como veremos más adelante, esta escucha y corrección pasan a ser completamente autónomas.

\subsubsection{Corrección del propio discurso motivado por el discurso de un Otro que señala la incorrección}

El fragmento $V I$ corresponde a una clase particular de $\mathrm{N}$ en mayo de 2020. En este fragmento la estudiante leía la producción escrita que había realizado como tarea. Reproducimos un fragmento de dicho texto:

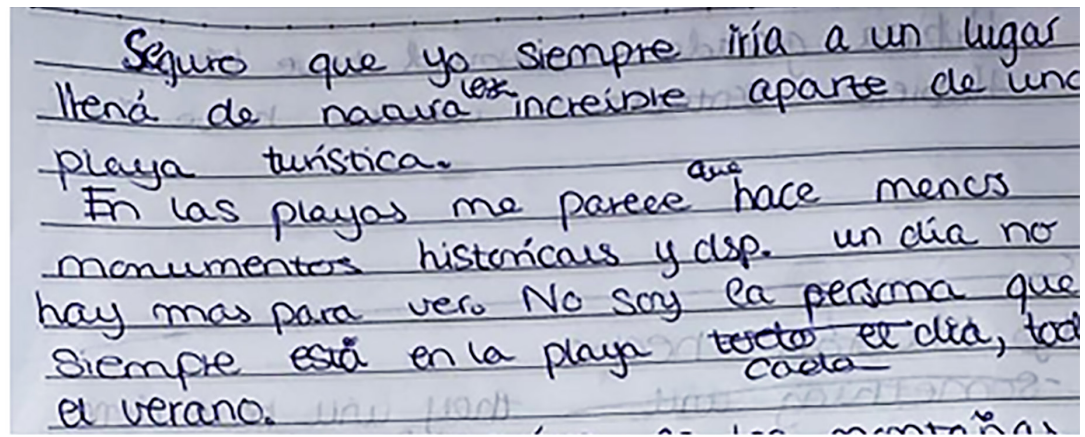

Imagen 2. $\mathrm{N}$ fragmento de escritura

La profesora anotaba los "errores" mientras $\mathrm{N}$ leía. Luego de la lectura, la profesora señaló el uso de determinada forma verbal, "hace menos monumentos históricos", a lo que devino la siguiente situación:
$\mathrm{N}: m m m$ (?) / dónde (?) hace menos monumentos históricos / $\mathrm{mmm}(?)$ ah no tiene sentido
Profesora: ajá
$\mathrm{N}$ : mmm quería decir / que no está (?) monumentos
Profesora: hay
$\mathrm{N}$ : hay (!) por supuesto
(Fragmento VI - 04052020)

Profesora: al principio dijiste algo de en las playas hace menos monumentos

$\mathrm{N}$ : -completamente confundida buscó en su texto esa frase y leyó- en las playas me parece que

Cuando la profesora repite, vemos que se produce un extrañamiento en $\mathrm{N}$; al volver a leerlo, el extrañamiento se refuerza y se produce la escucha (Bruzzo y Wingeyer, 2020a y 2020b). Tal como apunta Lemos (2002), retomando a Freud, el sujeto que habla y el que escucha son dos distintos, así N pudo 
escucharse y reformular su discurso. Aunque la estudiante no dio con la forma correcta, sí reformuló la parte problemática, el verbo "hacer" fue modificado por "estar". Lo que hace a esta situación, entonces, de la tercera posición es el hecho de que reconoció la diferencia entre la expresión sustituida y la que la sustituyó, frente a las similitudes entre ellas; a esto Lemos lo considera como "efecto contrario a los procesos metafóricos” (2002, p. 62), procesos metafóricos que eran característicos de la posición anterior.

\subsubsection{Corrección con indicación indirecta del Otro}

El fragmento VII corresponde a una clase particular de $\mathrm{N}$ en junio de 2020, en la que se estaba desarrollando una actividad oral: mediante preguntas abiertas personales, se motivaba a la aprendiente a recurrir a estructuras del subjuntivo.

$\mathrm{N}$ : si pudiera cambiar algo de lo que pasó hoy (?) $\mathrm{mm} /$ proteger mis piernas de la pintura / tiene sentido eso (?)

$\mathrm{V}: \mathrm{mm}$ sí el contenido está bien pero la parte gramatical tiene algo raro

$\mathrm{N}$ : AH sí vuelvo a intentarlo / si pudiera cambiar algo de lo que pasó hoy hubiera protegido mis piernas de la pintura

(Fragmento VII - 11062020)

Lo que hace que esta muestra breve sea considerada como de la tercera posición es el hecho de que, en primer lugar, la alumna al preguntar "tiene sentido eso (?)" ya da indicios de una escucha y, en segundo lugar, cuando la profesora le indica que había algo para corregir desde lo estructural, $\mathrm{N}$ puede corregir exitosamente su enunciado: "proteger mis piernas" pasa a "bubiera protegido mis piernas".

\subsubsection{Corrección del propio discurso motivado por su propia escucha}

Los fragmentos VIII y $I X$ corresponden a situaciones en las que el sujeto se corrige a sí mismo sin necesidad de intervención del Otro. En ambos casos, $\mathrm{K}$, en el fragmento VIII, y $\mathrm{N}$, en el fragmento $I X$, fueron arrastradas por el devenir del discurso -al igual que algunos casos que Desinano (2009) señala en la escritura del estudiante universitario-y emergen cadenas de significantes que no concuerdan con las estructuras correspondientes en español, y ambas se escuchan en el momento y se corrigen.

E1 fragmento VIII fue extraído de una clase en noviembre del curso en la UNNE en la que K comentaba una anécdota de un examen en la Facultad de Ingeniería de la UNNE:

K: cuando yo hice la parcial / eeeh yo estaba así esperando más hojas

Profesora: ah eso sí no

$+++$

$\mathrm{K}:+$ nosotros que tenemos que sacar así yo pensé eso es muy bueno para quien / cola (?) / cómo

I cómo es cola (?)

$\mathrm{J}$ : aam quien copia (?)

K: tipo alguien que hace así tipo hace una consulta un apunte y saca sus hojas y yo aaah (!)

(Fragmento VIII - 07112019)

\footnotetext{
6. Cabe destacar que el lenguaje no verbal cumplía una función importante en la construcción de sentido en esta situación,
} ya que a través de gestos $\mathrm{K}$ construyó la situación que estaba explicando. 
K se escucha en dos ocasiones: en la primera nota que "cola" no pertenece al español, pide a su compañera la traducción y, aun habiéndole su compañera indicado lo requerido, explica, tanto verbalmente como a través del lenguaje no verbal, la situación de "copia" a la que se estaba refiriendo. La segunda situación se da cuando dice "consulta" que la reemplaza por "apunte".

El fragmento $I X$ pertenece a una clase en julio, $\mathrm{N}$ estaba comentando las actividades de tarea que realizó y las que no.

$\mathrm{N}$ : Como / sentía que también tenía otra tarea pero no podría podía buscar la otra otro ejercicio que el tres y el cuatro / el tres y / el buscando justicia y leí un poquito del texto

$\mathrm{V}$ : bien sí era el tres el que dice completá con

$\mathrm{N}$ : sí lo hech lo he hecho

(Fragmento IX - 07072020)

Este caso es especialmente característico del arrastre por el devenir del discurso. Las estructuras reemplazadas son sumamente similares: (i) “podría”y "podía”, y"lo hecho"y "lo he hecho" por sus cercanías fonéticas y (ii) "otra" y "otro" por ser el mismo lexema con variación morfológica de género. En todos los casos, $\mathrm{N}$ se pudo corregir acertadamente debido a la escucha de la diferencia frente a la similitud.

\subsubsection{Reconocimiento de la ironía, el chiste, el doble sentido, los juegos de palabras}

El fragmento $X$ fue extraído de una clase durante junio de 2020, en la que N y la profesora estaban hablando sobre frases hechas para expresar sorpresa. La profesora le comentó algunas frases ya que $\mathrm{N}$ no recordaba otras, a lo que se dio la siguiente situación:

Profesora: no lo puedo creer (!)

$\mathrm{N}: \mathrm{mmm}$

Profesora: en serio (?)

$\mathrm{N}$ : en serio (?) / -risas-

Profesora: y después tenemos esas palabras que les decimos malas palabras / están las argentinas como qué mierda (?!)

$\mathrm{N}$ : qué mierda sí (!) / qué caja (!) en la casa de papel dicen todo el tiempo qué caja

Profesora: qué caja (?)

N: siíí

Profesora: ah mirá nunca lo escuché

$\mathrm{N}$ : -risas- no (?) / caja / viste la casa de papel (?)

Profesora: No / no vi la caja eh (!) la casa de papel -risas-

$\mathrm{N}$ : -risas- la caja de papel (!) -risas-

(Fragmento $X-23062020$ )

El reconocimiento del juego de palabras se da en dos ocasiones, en primer lugar, cuando $\mathrm{N}$ ríe al notar que cuando dijo "en serio (?)" dio la impresión de que se sorprendió por el hecho de que la expresión “¿en serio?” funciona para manifestar sorpresa, cuando en realidad la repetía, como solía hacerlo, como método mnemotécnico de frases, palabras o conceptos. En segundo lugar, en cuanto a la palabra "caja”, $\mathrm{N}$ notó fácilmente el juego de palabras producido por la semejanza fonética entre esa palabra y la palabra "casa". 


\section{A manera de cierre: continuaciones, profundización y aplicaciones en el ámbito de enseñanza}

Como venimos observando en los resultados que surgen de esta investigación, el fenómeno de la adquisición de una lengua extranjera, en nuestro caso, del español, puede muy bien ser entendido como un proceso de subjetivación. Esto significaría un modo de comprender la heterogeneidad en el proceso de adquisición diferente a los de los enfoques vigentes, ya que serían constitutivos de las relaciones que el sujeto establece con el lenguaje, el español como lengua extranjera en esta investigación; lengua que nunca llegará a dominar, sino más bien en la que se constituirá subjetivamente.

Para cerrar, cabe destacar que estos son los primeros resultados sobre las funciones de la interacción en el proceso de subjetivación. Queda para próximos trabajos analizar en mayor detalle estas situaciones tanto para determinar sus funciones como para precisar mayores detalles sobre cada posición y los momentos que las caracterizan.

Por último, consideramos que los resultados de esta investigación pueden ser útiles para el desarrollo de futuras propuestas para la enseñanza-aprendizaje de ELE, debido al cambio de perspectiva sobre el proceso de adquisición. Es por ello que, también nos proponemos a futuro observar y determinar, con mayor precisión, prácticas y estrategias de los docentes que en la interacción estimulen el proceso de subjetivación.

\section{Referencias bibliográficas}

Báez, M. (2000). Reflexión acerca de los rasgos lingüisticos de la interacción en la producción dialógica: nuevas perspectivas acerca del lenguaje oral. Trabajo Final de Seminario La pragmática comunicativa: análisis de textos conversacionales. Manuscrito no publicado.

Bruzzo, M.V.y Wingeyer, H. R. (2019). Análisis de expresiones escritas de dos estudiantes extranjeros en la UNNE: la constitución del sujeto en el discurso académico en ELE. X Congreso Nacional de Didáctica de la Lengua y la Literatura, San Martín, Argentina. Manuscrito no publicado.

Bruzzo, M. V. y Wingeyer, H. R. (2020a). La adquisición del español como lengua extranjera como proceso de subjetivación: una cuestión de captura. En Anais do XI Congresso Brasileiro de Hispanistas (pp. 1-15), Campina Grande, Realize Editora. https://bit.ly/3p0MBIm.

Bruzzo, M. V. y Wingeyer, H. R. (2020b). Adquisición del español como lengua extranjera como proceso de subjetivación. Estudio de caso. Contextos, 47, 1-27. https://bit.ly/3sHtdT2.

Camblong, A. M., Alarcón, R. y Di Módica, R. (2012). Alfabetización semiótica en las fronteras. Posadas, EDUNAM.

Desinano, N. (2009). Los alumnos universitarios y la escritura académica. Análisis de un problema. Rosario, Homo Sapiens. 
Desinano, N. (2018). La condición precaria del sujeto como hablante y la fragilidad del sistema de la lengua. Ponencia presentada en $21^{\circ}$ InPLA Intercambio de Pesquisas em Lingüistica Aplicada. https:// bit.ly/2YH2lo4.

Ellis, R. (2020). A short history of ASL: Where have we come from and where are we going? Language Teaching, First View, 1-16.

Lemos, C. de (1986). Interacionismo e Aquisição de Linguagem. DELTA, 2 (2), 231-248.

Lemos, C. de (2000a). Questioning the notion of development: the case of language acquisition. Culture E' Psychology, 6 (2), 169-182. https://bit.ly/3idkUcv.

Lemos, C. de (2000b). Desenvolvimento da linguagem e processo de subjetivação. Interações, 5 (10), 53-72. https://bit.ly/2G9RP37.

Lemos, C. de (2002). Das vicissitudes da fala da criança e de sua investigação. Cadernos de Estudos Lingüísticos, 42, 41-69.

Ortega, L. (2009). Understanding Second Language Acquisition. London, Routledge.

Wingeyer, H. R., Bruzzo, M. V. y Angelina, A. (2019). Expresiones escritas de estudiantes extranjeros de la UNNE. El proceso de constitución en sujeto del discurso académico. En Barroso, S. y Dandrea, F. (comps.), Actas X Coloquio CELU (pp. 101-114). Río Cuarto, Universidad Nacional de Río Cuarto.

Wingeyer, H. R. y Bruzzo, M. V. (2019). La escritura de estudiantes extranjeros de la Universidad Nacional del Nordeste (UNNE). Fragmentariedad y distorsiones enunciativas. Itinerarios Educativos, 12, 35-50. https://bit.ly/38oQKg6.

*María Virginia Bruzzo es Licenciada en Letras por la Universidad Nacional del Nordeste. Es Becaria de Iniciación (Secretaría General de Ciencia y Técnica de la UNNE) con funciones en el Instituto de Letras. Además, es Auxiliar Docente en Taller de Comprensión y Producción de Textos en la Facultad de Humanidades de la misma universidad. Su investigación se centra en la adquisición del español como lengua extranjera.

RECIBIDO: $1 / 12 / 2020$

ACEPTADO: 29/12/2020 